\title{
Neutron scattering from graphene oxide paper and thermally exfoliated reduced graphene oxide
}

\author{
E. F. Sheka ${ }^{1}$, I. Natkaniec ${ }^{2}$, V. Mel'nikov ${ }^{3}$, K. Druzbicki ${ }^{2,4}$ \\ ${ }^{1}$ Peoples' Friendship University of Russia, Miklukho-Maklaya Str. 6, 117198 Moscow, Russia \\ ${ }^{2}$ Faculty of Physics, Adam Mickiewicz University, Umultowska Str. 85, 61-614 Poznań, Poland \\ ${ }^{3}$ Semenov Institute of Chemical Physics RAS, Kosyguina str. 4, 119991 Moscow, Russia \\ ${ }^{4}$ Frank Laboratory of Neutron Physics, Joint Institute for Nuclear Research, \\ Joliot-Curie Str. 6, 141980 Dubna, Russia \\ sheka@icp.ac.ru,inat@amu.edu.pl,melnikov@center.chph.ras.ru
}

\section{PACS 61.05.fg; 61.46.+w; 63.22. $\pm \mathrm{m} ;$ 81.05.Uw \\ DOI 10.17586/2220-8054-2015-6-3-378-393}

Synthetic graphene oxide, in the form of graphene oxide paper (GOpp), and its reduction product - thermally exfoliated reduced graphene oxide (TErGO) - were studied by elastic and inelastic neutron scattering at low and room temperature conditions. The neutron diffraction patterns were analyzed to confirm stacking structures of both species consisting of $4-6$ and $\sim 8$ layers of microsize lateral dimension and the interlayer distances of $7.21 \AA$ and $3.36 \AA$, respectively. The one-phonon hydrogen amplitude-weighted density of vibrational states $G(\omega)$ represents the inelastic incoherent neutron scattering spectra of the products. The study has revealed the retained water in the freshly made GOpp, corresponding to the lowest humidity. The analysis of the TErGO $G(\omega)$ spectrum has disclosed the chemical composition of its circumference attributing the latter to sets of $\mathrm{CH}$ units with a minor presence of atomic oxygen.

Keywords: Carbon nanostructures, graphene oxide, reduced graphene oxide, neutron diffraction, inelastic neutron scattering.

Received: 18 March 2015

\section{Introduction}

By the definition of K. Novoselov et al. graphene modern technology can be divided into two independent domains, namely: 'low performance' (LP) and 'high performance' (HP) [1]. The former includes a wide range of practical applications based on nanoscale graphene material. Typical products of this domain are touch screens, electronic paper, foldable organic light emitting diodes and others. Products of the second domain, based on a micro-size and larger single or multi-graphene sheets, concern electronic device such as a high-frequency, logic and thin-film field transistors. This separation of the graphene technology into two types, held de facto, is not just a tribute to simplify operating with complex technologies, but, in fact, is a consequence of the nature of the graphene molecule-crystal dualism and technical implementation of its unique chemical and physical properties [2], respectively. Both the successful implementation of any technology and the difficulties arising on this way depend primarily on the material in use. Serious problems associated with the development of processing methods aimed at the mass production of micro- and macro-dimension crystalline graphene sheet are objective reasons for postponing the implementation of the graphene HP technology up to $2030[1,3]$. The latter is further exacerbated by the very high cost of this material [4]. Implementation of the LP technology has proved more successful. Active efforts of numerous teams of chemists 
have resulted in solving the problem of mass production of the necessary technological material - technical (molecular) graphene. This material is the final product of a complicated chemicaltechnological cycle, a concise description of which is shown in Scheme 1. In numerous cases, structural analysis shows pronounced non-planarity for GO sheets and nearly complete recovery of the planarity for the basal plane of the carbon skeleton in reduced graphene oxide (rGO) sheets, in connection with which thus produced rGO is referred to as graphene.

\begin{tabular}{|c|c|c|c|c|}
\hline Dispersin & $\rightarrow$ & $\begin{array}{l}\text { Oxidation of dispersed } \\
\text { graphite and producing } \\
\text { graphene oxide }(\mathrm{GO})\end{array}$ & $\rightarrow$ & $\begin{array}{l}\text { Reduction of GO and } \\
\text { producing reduced } \\
\text { graphene oxide (rGO) }\end{array}$ \\
\hline
\end{tabular}

\section{SCHEME 1}

Unlike hitherto used technological materials of strictly standardized chemical composition and structure, such a standardization of both GO and rGO products is not possible. The point is that the two terms refer to very wide ranges of substances, which actually represent various oxyhydride polyderivatives of graphene molecules of different chemical composition, size, and shape due to which the products can be characterized only by a restricted number of common features. Thus, in the GO case, there are two such characteristics that concern 1) interlayer distance between the GO sheets of $\geq 7 \AA$ and 2) an average chemical composition described by the $\mathrm{C}: \mathrm{O} \sim 2: 1$ ratio. The former exhibits the fact that both basal plane and the circumference area of the parent graphene molecules are involved in the oxidation while the latter points to the prepotency of epoxy units among the GO oxygen-containing groups (OCGs). A common feature of $\mathrm{rGO}$ is related to the interlayer distance of $\sim 3.5 \AA$ that points to the recovered planarity of the rGO carbon skeleton, on one hand, and to the location of residual OCGs in the circumference area $[2,5]$, on the other. Individual differences within the two communities are caused by the difference in size and shape of carbon skeletons of the parent graphene molecules and, consequently, by different numbers of attached OCGs, as well as by evidently individual combinations of these groups in each case. These circumstances result in that the structure and chemical composition of both GO and rGO may change on each stage of their chemical synthesis. The high-degree polyvariance of the chemical composition and structure of synthesized GO and rGO are widely discussed in the literature [6,7]. Thus, such an important parameter of rGOs produced in the different laboratories of the world as the residual oxygen concentration can vary up to 20 times.

The lack of uniform properties deprives the obtained synthetic products the requested technological stability and stimulates studies aimed at both disclosing the sources of nonuniformity and finding the way to produce reliably standard products. Even now, the question of GO and rGO chemical composition is a major stumbling block toward technical progress. Empirical solution of the problem rests mainly in determination of $\mathrm{C}: \mathrm{O}: \mathrm{H}$ mass contents of the products. While the determination requires the application of a set of complementary techniques, e.g. XRF, TGA, Raman and FTIR spectroscopy, XRD, XPS, REELS, and so forth, it is mainly focused on the evaluation of carbon and oxygen contents, neglecting hydrogen and other small dopants. However, as was shown recently [8], elucidation of hydrogen's role permits one to come close to addressing the key question of chemical composition for both GO and rGO products. The quoted results were obtained by studying the scattering of thermal neutrons that is extremely sensitive to protium. Once stimulated by the results obtained, the authors (I. N. and E. Sh.) have suggested to extend the neutron scattering study to a set of synthetic GO and rGO products of different origins. The performed study not only revealed the retained water and hydrogen-enriched cores of the products as new facets of the product structure and chemical 
composition, but also to show the way to link differences in the product behavior with the chemical protocols of their production as well as to shed light on the reasons of their instability over time. Recently, such a study has been performed for chemically produced GO/rGO pair (Ak-GO and Ak-rGO below) [9] provided by the Akkolab company [10]. The current paper presents the neutron scattering studies of chemically produced GO as GO paper and thermally exfoliated rGO (ppGO and TerGO below).

\section{Methods in use}

\subsection{Sample preparation}

Graphite oxide (GrO) and GO paper. The samples of oxidized graphite were prepared according to the Hummers technique [11]. In the standard procedure, $20 \mathrm{~g}$ of particulate pencil graphite (ash $<1 \%$ ) were charged into a solution of $10 \mathrm{~g}$ of $\mathrm{NaNO}_{3}$ in $460 \mathrm{ml}$ of $94 \% \mathrm{H}_{2} \mathrm{SO}_{4}$ and stirred for one hour at room temperature. Then the reactor was cooled to $0{ }^{\circ} \mathrm{C}$ and $60 \mathrm{~g}$ of $\mathrm{KMnO}_{4}$ were added to the suspension in small portions under stirring. After introduction of the permanganate, the reactor temperature was raised to $35 \pm 3{ }^{\circ} \mathrm{C}$ and has been maintained for one hour. Upon reaction completion, $920 \mathrm{ml}$ of water were slowly added to the mixture and the temperature was raised to $\sim 100{ }^{\circ} \mathrm{C}$. The temperature was maintained for $15 \mathrm{~min}$, after which the mixture was diluted with $1400 \mathrm{ml}$ of water and treated with hydrogen peroxide $(3 \%, 200 \mathrm{ml})$ to convert manganese dioxide to soluble and colorless manganese sulfate. GrO was allowed to settle and residual slightly yellow acidic solution was removed. The obtained $\mathrm{GrO}$ was repeatedly redispersed and centrifuged in $\mathrm{HCl}(1 \%)$ solution at first until sulfate ions were removed and then in distilled water until the GO paste obtained displayed neutral $\mathrm{pH}$. The paste was placed on a polytetrafluoroethylene plate and dried at room temperature to give free standing GO paper (ppGO below) (average thickness of $20-30$ microns). Sheets of ppGO were cut into pieces of $10-15 \times 40-50 \mathrm{~mm}^{2}$, dried at $60{ }^{\circ} \mathrm{C}$ overnight and stored in a sealed container. The general appearance of the ppGO is presented in Fig. 1a. Scanning electron microscopy was performed on a JSM7001F microscope. An accelerating voltage of $3-15 \mathrm{kV}$ was used for observations. Images were obtained in the secondary electron mode.

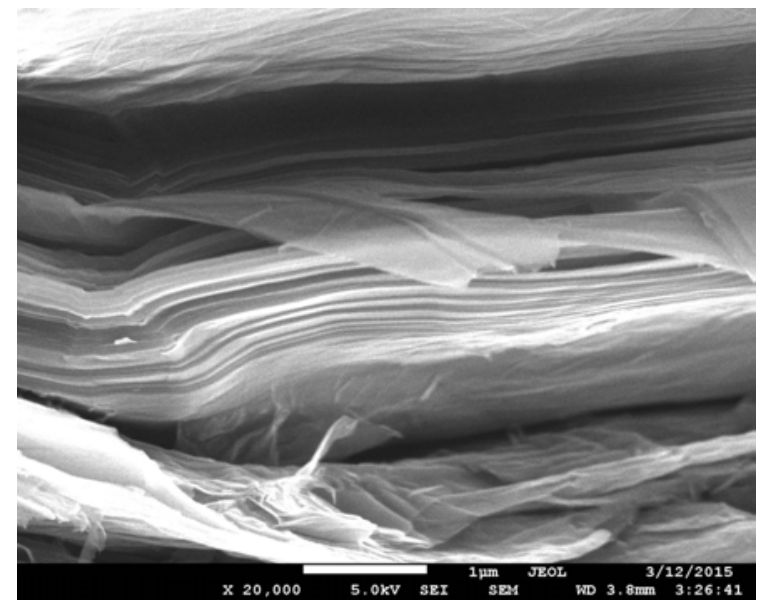

a)

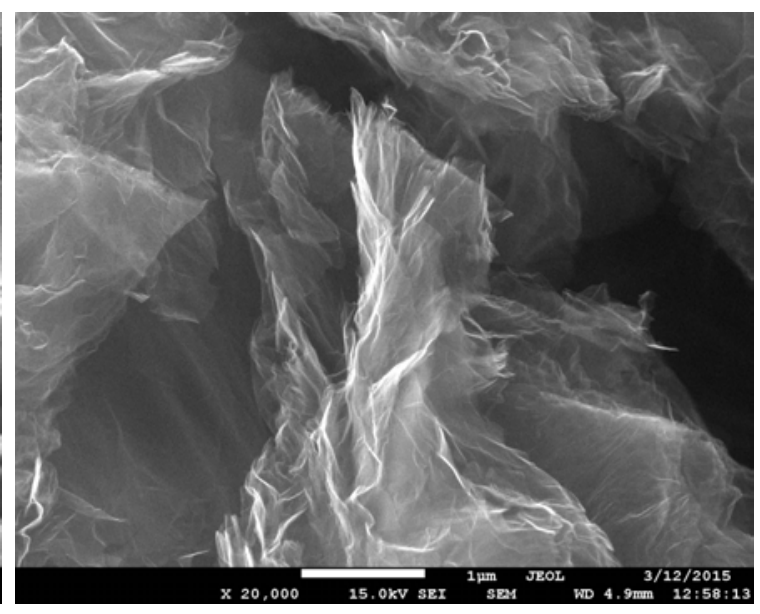

b)

FIG. 1. SEM images of ppGO (a) and TErGO (b). White bars mark the image scaling of $1 \mu$ in both panels 
TABLE 1. Mass content of ppGO and TErGO according to gaseous volumetry (gv) and elemental analysis (ea), wt \%

\begin{tabular}{c|c|c|c} 
Sample & $\mathrm{C}$ & $\mathrm{H}$ & $\mathrm{O}^{1}$ \\
\hline ppGO (gv) & 56.2 & 0.7 & 40.5 \\
\hline TErGO (ea) & 85.4 & 0.5 & $14.1^{2}$ \\
\hline TErGO (gv) & 87.1 & 0.5 & $12.1^{2}$
\end{tabular}

${ }^{1}$ Oxygen content was obtained as the difference between total sample weight and the sum of weights related to other elements.

2 Presumably, the contact of highly active TErGO with air is mainly responsible for the oxygen content as well as for water.

Thermally exfoliated $\boldsymbol{r G O}$. Thermal shock is widely used to produce thermally expanded graphite, as well as exfoliation and/or reduction of GO [12]. This type of thermal treatment initiates the exothermic decomposition of oxygen containing groups (OCGs) that is accompanied by expansion due to gas evolution. In the current study, thermally reduced graphene oxide (TerGO) was obtained by introducing ppGO into a preheated $750{ }^{\circ} \mathrm{C}$ tubular furnace. As a result of the exothermic reduction reaction, which was accompanied by gas evolution and a rapid splitting of the layered ppGO structure, the TerGO sample obtained had a specific surface area of $500-600 \mathrm{~m}^{2} / \mathrm{g}$. The freshly-prepared fluffy and voluminous TErGO was dispersed in water, compacted by vacuum filtration to reduce volume and dried at $100{ }^{\circ} \mathrm{C}$. The general appearance of the TErGO is presented in Fig. 1b.

Characterization methods. The element content $(\mathrm{C}, \mathrm{H}$, and $\mathrm{O})$ of both ppGO and TErGO was determined in due course of elemental analysis and gaseous volumetry. The former was performed with PE $2400 \mathrm{CHN}$ Analyzer (Perkin Elmer, USA), which employs a combustion to convert the sample elements to $\mathrm{CO}_{2}, \mathrm{H}_{2} \mathrm{O}$ and $\mathrm{N}_{2}$ gases. The gaseous volumentary monitors the yields of gaseous products $\left(\mathrm{CO}_{2}, \mathrm{CO}, \mathrm{H}_{2} \mathrm{O}\right.$, and other) under stepwise annealing of the samples at different temperature. Typical temperature dependences of the measured yields for ppGO and TErGO samples are presented in Fig. 2. The accuracy of each element determination was about $0.30 \mathrm{wt} \%$ in both cases. Table 1 summarizes data on mass content of the studied samples.

Both ppGO and TErGO were analyzed by X-ray powder diffraction (XRD). Data collection was done with DRON-3M diffractometer ( $\mathrm{CuK} \alpha$ radiation). Diffraction patterns were collected between $8^{\circ}$ and $50^{\circ}$ of $2 \theta$. The XRD patterns of ppGO consisted of characteristic intense peaks positioned at 7.6 - 7.2 $\AA$ sometimes followed with peaks of unreacted graphite at $3.36-3.38 \AA$. The variation of the main peak positions is due to different content of retained water [13] the quantity of which is highly sensitive to details of the sample treatment. The XRD patterns of TErGO consisted of single peaks at $3.36 \AA$.

\subsection{Neutron Scattering Experiments}

The neutron scattering study was performed at the high flux pulsed IBR-2 reactor of the Frank Laboratory of Neutron Physics at the Joint Institute for Nuclear Research by using the inverted-geometry spectrometer NERA [14]. The investigated samples were illuminated by a white neutron beam analyzed by time-of-flight method on the $110 \mathrm{~m}$ flight path from the IBR-2 moderator to the sample. The NERA spectrometer allows for simultaneous recording of both neutron powder diffraction (NPD) and inelastic neutron scattering (INS) spectra. The NPD spectra were obtained for two scattering angles of diffraction detectors, the lowest and the highest of which allows one to measure the inter-planar distances up to $9 \AA$ and $4 \AA$, 

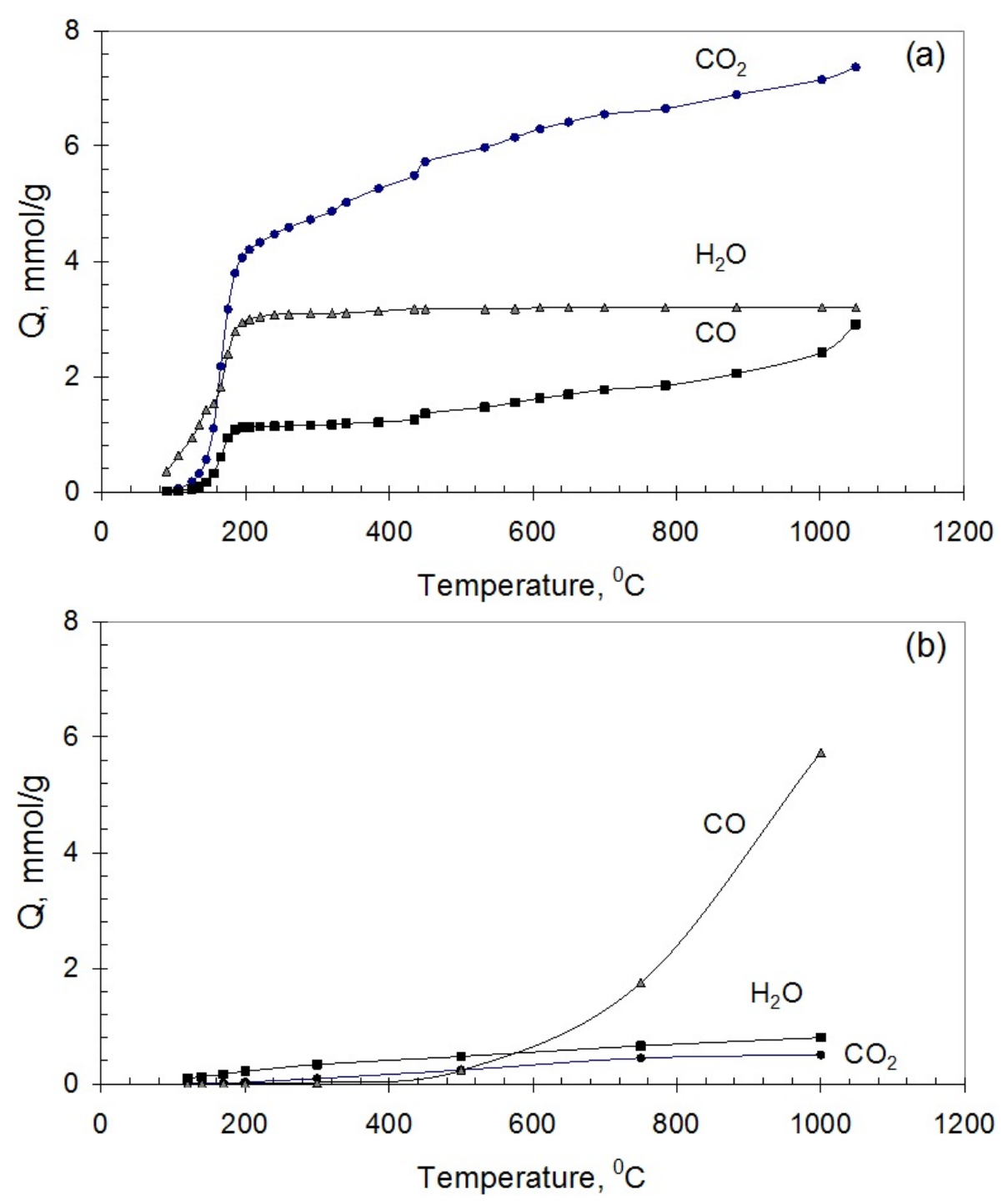

FIG. 2. Temperature dependence of the yields of the evolved gases $\mathrm{Q}$ at stepwise annealing of ppGO (a) and TErGO (b)

respectively. The INS spectra were collected at the final energy of the scattered neutrons, fixed by beryllium filters and crystal analyzers at $E_{f}=4.65 \mathrm{meV}$, over fifteen scattering angles from $20^{\circ}$ to $160^{\circ}$ by step of $10^{\circ}$. The experiments were performed at temperatures $6 \mathrm{~K}$ and $295 \mathrm{~K}$.

\section{Experimental Results}

\subsection{Neutron Diffraction}

Figure 3 presents a set of the NPD plots, recorded at $6 \mathrm{~K}$ for ppGO and TErGO samples, alongside with the ones related to spectral graphite, which serves as the reference, collected at two different angles. As seen in Fig. 3a, the diffraction at $2 \Theta=44.7^{\circ}$ provides a good vision of the structural features over $3 \AA$, while the diffraction at $2 \Theta=117.4^{\circ}$ (see Fig. 3b) allows tracking the sample structure in the region from $1 \AA$ to $2.5 \AA$. The main feature of the ppGO found in Fig. 3a is described by a broad peak at $7.21 \AA$ that is equivalent to the $\operatorname{Gr}(001)$ reflex forbidden in graphite structure and gives an evidence for a stacked ppGO structure with the relevant interlayer distance. The peak is noticeably broadened (FWHM constitutes $0.5 \AA$ ) 

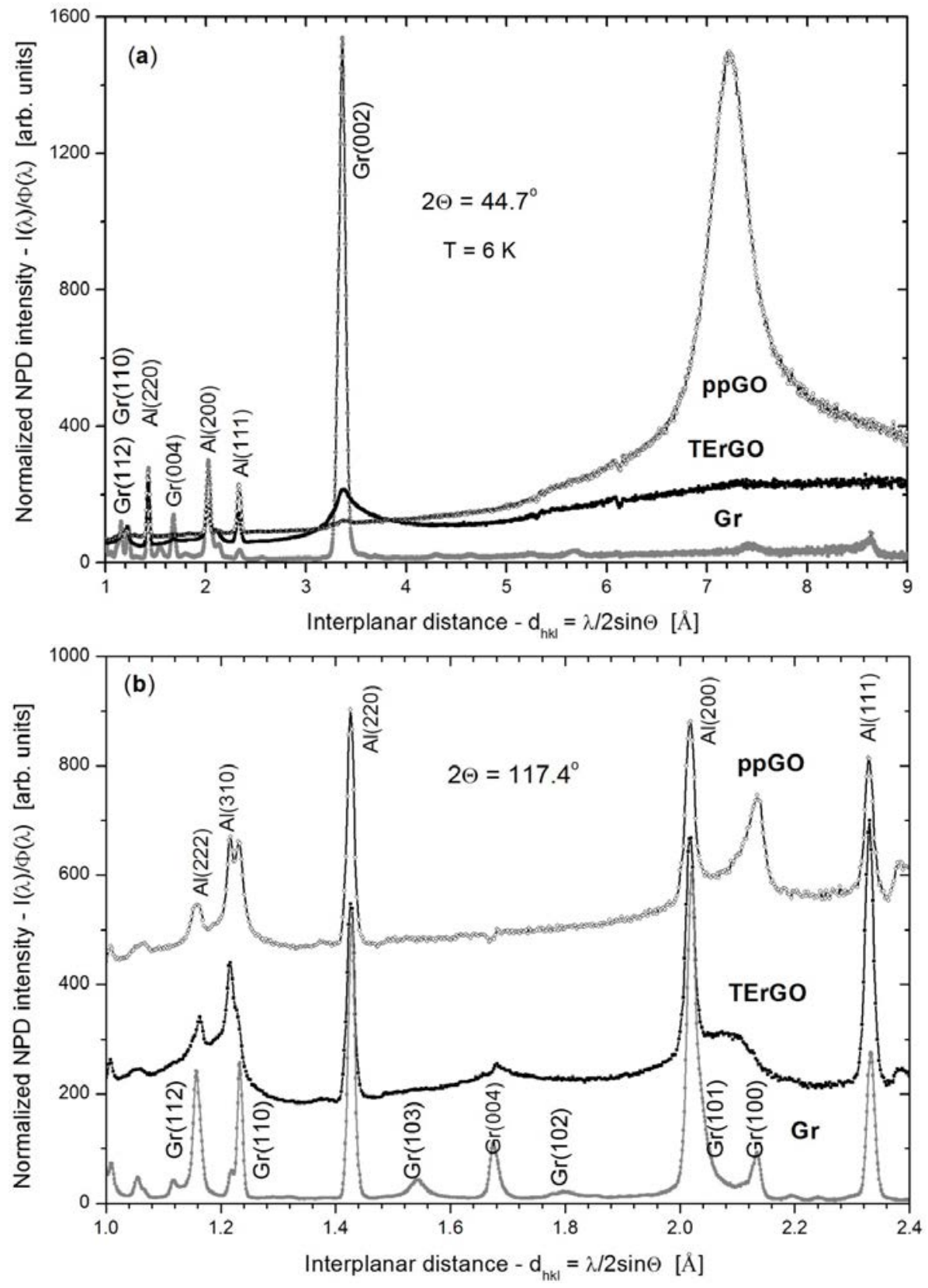

FIG. 3. NPD of spectral graphite (Gr), graphene oxide paper (ppGO) and thermally exfoliated reduced graphene oxide (TErGO) recorded at $T=6 \mathrm{~K}$ at scattering angles $2 \Theta=44.7^{\circ}$ (a) and $2 \Theta=117.4^{\circ}$ (b). The data are normalized per neutron flux intensity $\Phi(\lambda)$ at each neutron wavelength $\lambda$; $\operatorname{Gr}(\mathrm{hkl})$ and $\mathrm{Al}(\mathrm{hkl})$ denote characteristic diffraction reflexes of spectral graphite and aluminum of cryostat and sample holder at different Miller indexes, respectively

with respect to the reference $\operatorname{Gr}(002)$ peak of graphite (FWHM of $0.034 \AA$ ), which exhibits a considerable size limiting of the stacks normal to the ppGO layers.

According to widely used Scherrer's equation, the FWHM of a diffraction peak $B$ and the corresponding coherent scattering region (CSR) length $L_{C S R}$ are inversely connected: $B=k \lambda / L_{C S R} \sin \Theta$, where $k$ is a fitting factor, $\lambda$ and $\Theta$ are the neutron wavelength and scattering angle, respectively. When the diffraction study for a set of samples is performed 
under the same conditions, it is possible to take one of the samples as the reference and to determine $L_{C S R}$ of the remaining samples. In our study, $L_{C S R}^{c^{r e f}}$ is attributed to the crystalline graphite and constitutes $\sim 20 \mathrm{~nm}$ along the $c$ direction [15]. Therefore, $L_{C S R}$ of the studied GO can be determined as:

$$
L_{C S R}^{c}=\left(B_{002}^{r e f} / B\right)\left(\lambda / \lambda^{r e f}\right) L_{C S R}^{c^{r e f}}
$$

Substituting $\lambda / \lambda^{r e f}$ by the ratio of the relative peak positions and using $B$ values given above, we obtain $L_{C S R}^{c}=2.9 \mathrm{~nm}$. Assuming that the thickness of a GO layer is $\sim 0.6 \mathrm{~nm}$ [16], the ppGO stacks may consist of $4-6$ layers.

The lateral dimension of the stacks is described by peaks found below $2.5 \AA$. The $\operatorname{Gr}(110)$ peak is particularly characteristic for graphite-like structures [17]. As seen in Fig. 3b, there are no ppGO peaks broader than the reference graphite ones in the region. This means that the corresponding $L_{C S R}^{a}$ values for graphite and ppGO are quite equivalent, which means the lateral dimensions of the GO stacks should evidently be larger than $20 \mathrm{~nm}$.

Similar analysis of the $\operatorname{Gr}(002)$ peak of TErGO in Fig. 3a (positioned at $3.36 \AA$ with FWHM of $0.26 \AA$ ) results in a $L_{C S R}^{c}$ value of $2.6 \mathrm{~nm}$, thus indicating $\sim 8$ layer stacks for the studied TErGO sample. Similar to the ppGO, none of the diffraction peaks is broadened (see Fig. 3b) in the region below $2.5 \AA$, which points to large TErGO stacks in the lateral dimension. The obtained data for both ppGO and TErGO samples are in good agreement with results from XRD studies, indicating submicron-micron lateral size for both ppGO and TErGO. The described structures are typical for $\mathrm{GO}$ and $\mathrm{rGO}$ of different origins, which has been supported by both our study of Ak-GO/Ak-rGO pair [9] and by the recent neutron diffraction study of Pumera and co-workers [18]. Raising the temperature to $295 \mathrm{~K}$ influences the described diffraction patterns only slightly.

\subsection{Inelastic Neutron Scattering}

Figure 4 presents the time-of-flight (TOF) INS spectra of the studied samples, recorded at $T=6 \mathrm{~K}$ and $295 \mathrm{~K}$. The spectra are summed over 15 scattering angles, normalized per 10 hours of exposure time and for the $10 \mathrm{~g}$ mass. Previous studies [9] found that the INS intensity of the same mass of graphite is so weak that it cannot be distinguished at the level of the instrumental background. In the current study, the latter was extracted from the sample spectra, thereby excluding the contribution of the scattering from carbon atoms as well. Since the chemical content of the samples includes only oxygen and hydrogen atoms beside carbons, the scattering should be attributed to the hydrogens since the neutron scattering ability of oxygen is similar to that of carbon. As seen in the figure, the ppGO sample scatters quite intensely, thus indicating it is hydrogen-enriched, while scattering from TErGO sample is much weaker. As follows from Fig. 2a, freshly prepared ppGO contains a significant amount of retained water that is obviously responsible for the sample INS. In contrast, the quantity of the retained water in the freshly made TErGO is much smaller (see Fig. 2b). A considerable evolution of water from the sample at $T>600{ }^{\circ} \mathrm{C}$ is evidently provided from the interaction of destructed TErGO with air. Since hydrogen atoms in retained water play the main role in the hydrophilic ppGO, its INS spectrum is, as expected, similar to that of oxygenated graphite (GrO) [13] and the previously studied AkGO [9]. The TErGO spectrum was quite different than that of retained water, thus the hydrogen atoms present are thought to be incorporated into the carbonaceous skeleton of the hydrophobic TErGO, similar to the chemically produced Ak-rGO [9] and natural rGO of shungite carbon (sh-rGO below) [8]. Altogether, the observed INS spectra of both samples are provided with incoherent inelastic neutron scattering (IINS) from hydrogen (protium) atoms. The temperature 
dependence is typical for molecular solids $[19,20]$ and is provided by considerable enhancement of multi-phonon scattering and a considerable smoothing of the total spectrum as a whole.

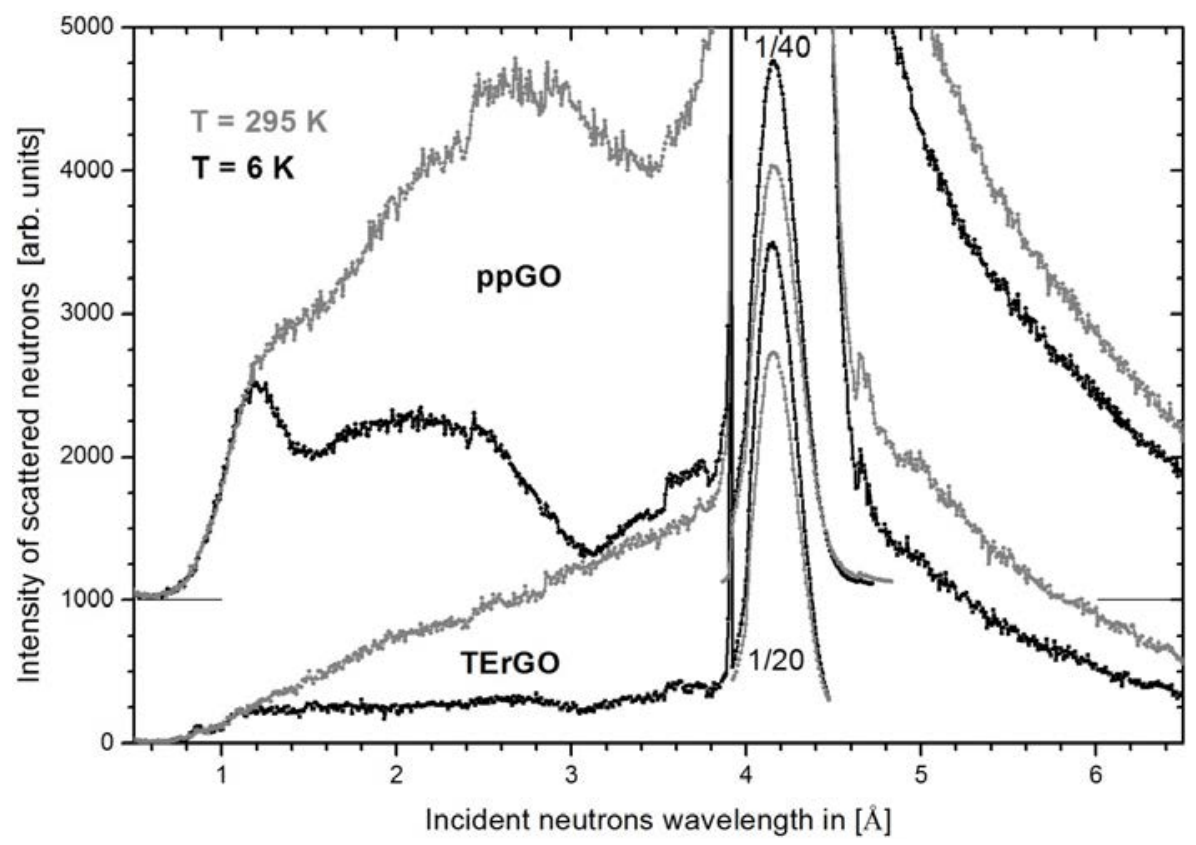

FIG. 4. Time-of-flight INS spectra of freshly made ppGO and TErGO samples, recorded at $T=6 \mathrm{~K}$ (black) and $295 \mathrm{~K}$ (light gray) normalized by monitor counter to the same number of incident thermal neutrons flux for the wavelength region $(0.8-6.8) \AA$, and exempted of background. The intensity of elastic peaks of the ppGO and TErGO spectra is 40 - and 20 -fold reduced, respectively

Within the confines of commonly used incoherent inelastic one-phonon scattering approximation, the IINS spectra intensity is determined by the scattering cross-section (see for example Ref. [19]) as:

$$
\sigma_{1}^{i n c}\left(E_{i}, E_{f}, \varphi, T\right) \approx \sqrt{\frac{E_{f}}{E_{i}}} \frac{\hbar\left|Q\left(E_{i}, E_{f}, \varphi\right)\right|^{2}}{\omega} \sum_{n} \frac{\left(b_{n}^{i n c}\right)^{2}}{M_{n}} \frac{\exp \left(-2 W_{n}\right)}{1-\exp \left(-\frac{\hbar \omega}{k_{B} T}\right)} G_{n}(\omega)
$$

Here, $Q\left(E_{i}, E_{f}, \varphi\right)$ is the neutron momentum transfer; $\omega=\left(E_{i}-E_{f}\right)$ is the neutron energy transfer; $b_{n}^{\text {inc }}$ and $M_{n}$ are the incoherent scattering length and mass of the $n$-th atom; $\exp \left(-2 W_{n}\right)$ is the Debye-Waller factor; $G_{n}(\omega)$ presents the $n$-th atom contribution into the amplitudeweighted density of vibrational states (AWDVS) expressed as:

$$
G(\omega)=\sum_{n} G_{n}(\omega)=\sum_{n} \sum_{j}\left[A_{j}^{n}(\omega)\right]^{2} \delta\left(\omega-\omega_{j}\right)
$$

Here, $A_{j}^{n}(\omega)$ is the $n$-th atom contribution into the eigenvector of the $j$-th phonon mode thus presenting the amplitude of the $n$-th atom displacement at the vibrational frequency $\omega_{j}$.

Figure 5 presents the one-phonon AWDVS $G(\omega)$ spectra obtained in the course of a standard treatment procedure [20] and related to the TOF spectra shown in Fig. 4. All the spectra are normalized per $10 \mathrm{~g}$ of mass. When obtaining $G(\omega)$ spectra, Debye-Waller factors were taken as unity, which meets the requirements at low temperature and rather narrow frequency region. The latter is met in the case of spectra at $6 \mathrm{~K}$ while spectra at room temperature 
are burdened by the temperature dependence of the Debay-Waller factors as well as by highly considerable contribution of multi-phonon scattering [19]. Only low-temperature $G(\omega)$ spectra will be considered later, since they present a suitable platform for testing $G(\omega)$ delivered by computational modeling of the structure and dynamics of the studied ppGO and TErGO. Due to different origins of the hydrogen scattering, let us consider the $G(\omega)$ spectra of the two samples separately.

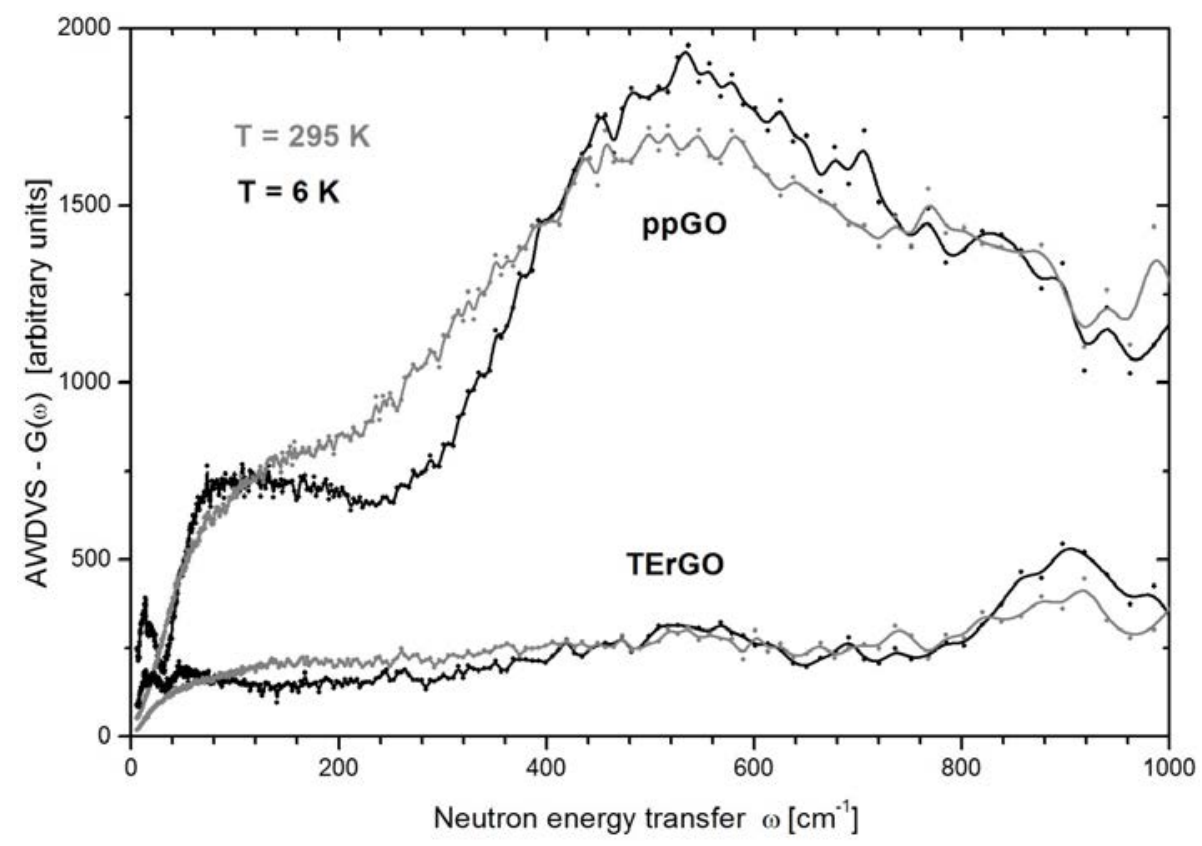

FIG. 5. Experimental amplitude-weighted density of vibrational states $G(\omega)$ spectra of ppGO and TErGO at $6 \mathrm{~K}$ (black) and $295 \mathrm{~K}$ (light gray) extracted from the TOF spectra in Fig. 4 under one-phonon approximation

\section{Discussion of Results}

\subsection{IINS Spectrum of the GO paper}

The $G(\omega)$ spectrum of ppGO at $6 \mathrm{~K}$, along with its temperature dependence, is similar to that observed for $\mathrm{GrO}$ at the lowest humidity conditions [13]. In our case, a characteristic interlayer distance of $7.21 \AA$ supports the conclusion. As was shown earlier, when the humidity becomes higher, the interlayer distance increases, but in a peculiar way. The four-fold lifting of the humidity from $20 \%$ to $80 \%$ stimulates a continuous growth of the interlayer spacing from 7.0 to $8.5 \AA$, while reaching $100 \%$ humidity causes an abrupt growth of the spacing to $11 \AA$. This anomaly is thought to arise because water is retained in $\mathrm{GrO}$ in the interlayer space, once bound with the GrO layers in the form of incomplete monolayer coverage at lower humidities while completing the monolayer covering at interlayer distance $8.0-10 \AA$ and forming additional water layer, which requires a sharp rise in the distance up to $11 \AA$ [13]. This conclusion has been repeatedly confirmed subsequently (see Refs. [21,22] and references therein), which gave a strong support in favor of the model suggested for calculations and received a strong support [9]. Let us consider the obtained results in the framework of the same model.

The left column of Fig. 6 presents $G(\omega)$ spectra of two GO samples, where the spectrum of the ppGO sample studied in the current paper is given in panel (a) while the second in 
panel (b) is related to the Ak-GO sample produced in [10] and previously studied in [9]. The model, which led the foundation for the description of the latter spectrum, is shown in Fig. 7. That involves two GO sheets separated by $7 \AA$ in average and 48 water molecules located on both sides of the sheets. The relevant $(5,5)$ GO molecule (Fig. 7a) is based on the parent $(5,5)$ graphene molecule (a rectangular fragment with five benzenoid units along armchair and zigzag edges). As has been shown, a molecule of this size is large enough to reliably reveal the main idiosyncrasies of graphene's chemistry $[5,23]$. It is currently widely accepted that the atomic oxygens and hydroxyls (mainly) and carboxyls (in minority) take part in the graphene oxygenation, resulting in the formation of epoxy and hydroxy groups on the molecule basal planes, while carbonyls, hydroxyls, and carboxyls are spread over the circumferences (see a detailed analysis in [5]). The distribution of these OCGs over the carbon core is not standard and may vary widely, depending on the chemical protocol in use as well as on external conditions of the oxygenation. The $(5,5) \mathrm{GO}$ molecule presented in Fig. 7a involves epoxy $(\times 11)$ and hydroxy $(\times 8)$ groups that are randomly spread over both sides of the basal plane (containing 44 carbon atoms), which corresponds to the average per-benzenoidring formula $\mathrm{C}_{6} \mathrm{O}_{2.5} \mathrm{H}_{1.2}$. The relevant formula, which follows from the atomic-mass-content of ppGO in Table 1, is $\mathrm{C}_{6} \mathrm{O}_{3} \mathrm{H}_{0.85}$, which is quite consistent with the model. Since the IINS cross-section of both carbon and oxygen atoms are about one order of magnitude less than that of protium, the contribution of the basal-plane atoms in the experimental spectrum is mainly provided due to the scattering from hydrogen atoms from hydroxyls.

In addition to the basal plane, the chemical composition of the GO periphery is of great importance. Usually, this area is presented by carbonyls, hydroxyls, and carboxyls, among which only two latter ones contribute to IINS. To make the periphery contribution more vivid, it was decided [9] to intentionally terminate the edge atoms with hydrogens. This termination gives one the potential to trace the changes of the IINS spectra of GOs after transformation into the rGOs, since the appearance of hydrogen atoms in the GO periphery always accompanies reduction (see detailed discussion in [8]).

Figure $7 \mathrm{~b}$ shows the double-layer 'solvated sandwich' of two $(5,5) \mathrm{GO}$ sheets surrounded by 48 water molecules. A detailed analysis has shown [9] that none of the water molecules is dissociated and each of them forms the hydrogen bonds (HBs) with the relevant GO layers (either $\mathrm{H} \cdots \mathrm{O}$ with epoxy groups or $\mathrm{O} \cdots \mathrm{H}$ with hydroxyls) that are supplemented with one-two HBs with neighboring water molecules. Evidently, the water molecules forming monolayers around $(5,5) \mathrm{GO}$ substrates are differently coordinated in terms of the number of H-bonds, $N_{H B}$. As occurred in the current model, $N_{H B}$ changes from 4 to 1 , thus pointing to four-, three-, two-, and one-HB configured molecules and constitutes 7, 10, 25, and 6 (7:10:25:6 HB-composition below), respectively. It should be noted that none of the four- and three-HB molecules surrounded by other water molecules, which is a characteristic feature of bulk water, is observed. Apparently, the dynamics of differently configured water molecules is not the same.

The right column panels in Fig. 6 presents the $N_{H B}$-partitioned spectra of water for $N_{H B}=1,2,3$, and 4 as normalized per $100 \%$ molecular fraction of each. As seen in the figure, differently $N_{H B}$-configured molecules provide quite differently shaped spectra. Hence, when turning from $N_{H B}=1$ to $N_{H B}=4$, the spectra undergo a continuous shifting toward higher wavenumbers. By comparing these spectra with the experimental $G(\omega)$ spectra of ppGO and AkGO presented in the left column of Fig. 6, one can readily see that differently $N_{H B}$-configured molecules contribute to different parts of the experimental spectrum. The water IINS spectrum has a characteristic two-humped look and consists of two bands located at $\sim 80 \mathrm{~cm}^{-1}$ (A) and $500 \mathrm{~cm}^{-1}$ (B) that are bridged with a smooth linking L. The spectrum attribution to particular vibrational modes is widely discussed and will not be considered here (see [9] and 

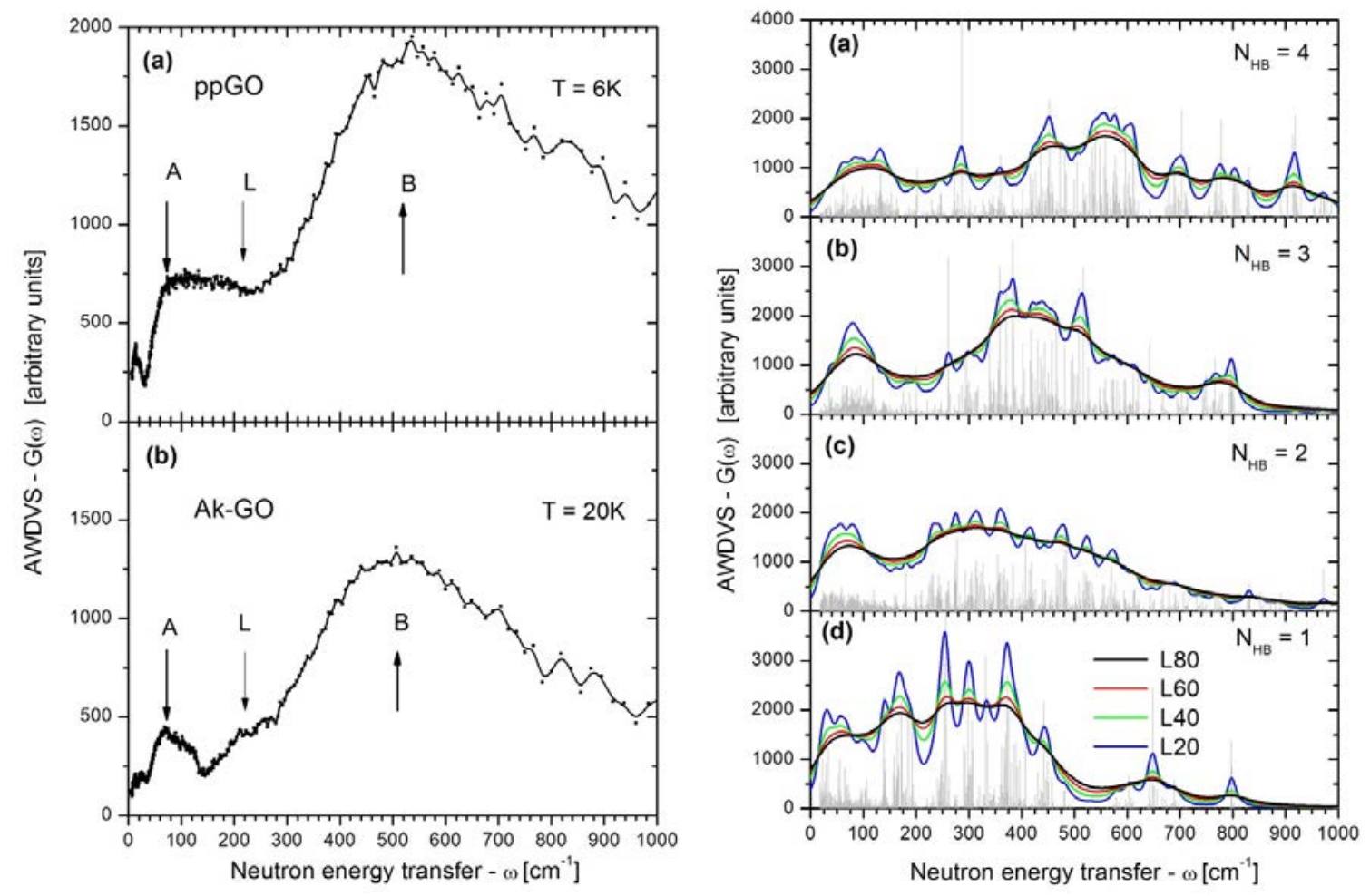

FIG. 6. AWDVS $G(\omega)$ spectra. Left: (a) Experimental $G(\omega)$ spectrum of ppGO at $6 \mathrm{~K}$; (b). The same as in panel (a) but related to Ak-GO at $20 \mathrm{~K}$ [9]. Right: $N_{H B}$-partitioned spectra of water molecules related to $N_{H B}=4$ (a); $N_{H B}=$ 3 (b); $N_{H B}=2$ (c); and $N_{H B}=1$ (d) [9]. Bar spectra intensities are normalized per the same number of water molecules each. The 0-1 IINS vibrational mode intensities (light gray bars) were convoluted by using Lorentzians of 20, 40, 60, and $80 \mathrm{~cm}^{-1}$ half width (black lines L20, L40, L60, and L80, respectively). The labeling is attributed to all panels
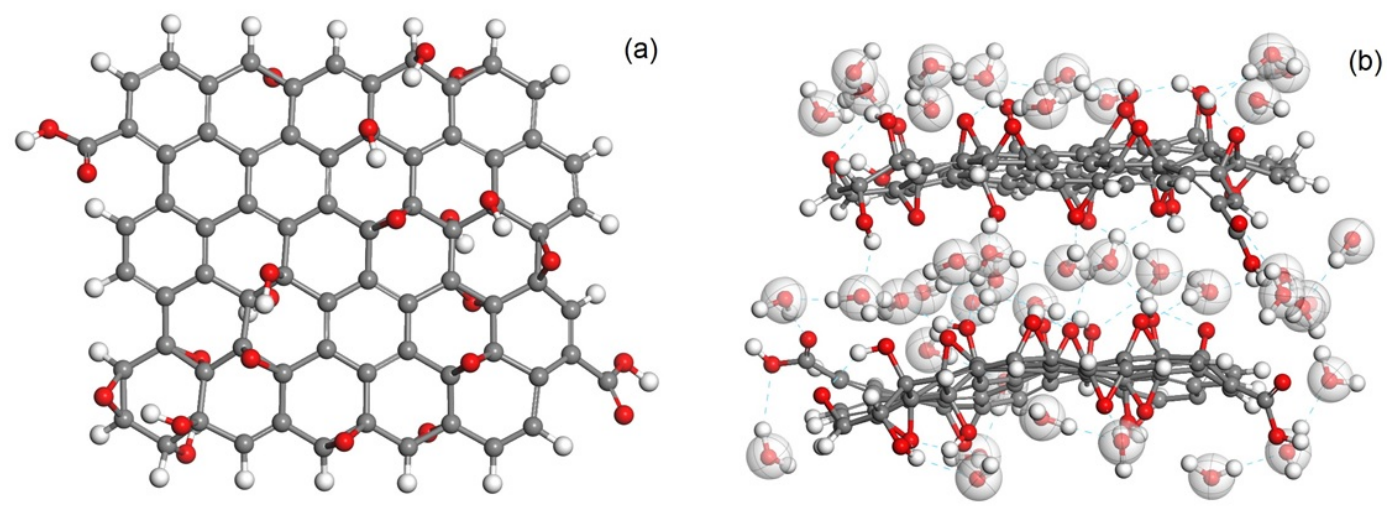

FIG. 7. Equilibrium structure of the $\{\mathrm{GO}+$ water $\}$ model [9]. (a). $(5,5) \mathrm{GO}$ molecule. (b). Two-layer solvated sandwich of $(5,5) \mathrm{GO}$ molecules including 48 water molecules (see detail description in [9]). Gray, red and white balls present carbon, oxygen, and hydrogen atoms, respectively 
references therein). As for calculated spectra, those related to $N_{H B}=1$ and $N_{H B}=2$ are mainly responsible for the A and $\mathrm{L}$ parts of the real spectrum while $N_{H B}=3$ and $N_{H B}=4$ spectra are consistent with the higher-wavenumber part B. Therefore, the experimental spectrum actually presents a convolution of the contributions provided with differently configured water molecules, which obviously depend on the relevant $N_{H B}$. In practice, the $N_{H B}$ composition is not only difficult to be predicted, but should not be treated as fixed, since the water is quite dynamic. Consequently, it is not surprising that the calculated 7:10:25:6 $N_{H B}$ composition of the $\{\mathrm{GO}+$ water $\}$ model [9] does not fit any of the presented experimental $G(\omega)$ spectra in detail. However, when all the molecules are artificially substituted by the $N_{H B}=4$-configured ones (panel (d)), fitting becomes much better, which might indicate the preferential presence of the $N_{H B}=4$ - configured molecules in the real case. The dependence of the total spectrum shape on the contribution of alternatively configured molecules may be the reason that the IINS spectra of the interlayer retained water are quite similar in different matrices with respect to the general character of the shape, while the position of B band - as well as intensity of the bridging linking $\mathrm{L}$ - are different due to a difference in the arrangement of water molecules in the two cases. The difference can be caused by both the varied chemical composition of the OCGs, which cover carbon skeletons, and size and shape of the skeletons themselves thus giving a topochemical character to the feature observed.

Particular attention should be given to the convolution series at each right panel of Fig. 6. According to Ex.(3), the originally calculated $G(\omega)$ spectra present the extended sets of $\delta$-functions. Multiple different internal and external factors, such as finite lifetime and anharmonicity of vibrations as well as various structural and dynamic inhomogeneities, result in the broadening of the spectral shape. This is usually taken into account via convolution of the $\delta$-spectra with Lorentzians with a different half-width L. As seen in the figure, the broadening significantly affects the spectra shapes, thus, the spectra related to L20 and L100 look differently. While L20 curves may be considered as the ones of the highest level of anharmonicity and short lifetime for vibrations, L100 curves disclose the inhomogeneity effects. The latter are highly expected in systems similar to the studied GO and rGO samples. A comparison of the spectra of both columns of Fig. 6 shows that not questioned inhomogeneity of the studied GO samples is rather significant and may be characterized in terms of Lorentzian's parameters by L80.

\subsection{IINS spectrum of TErGO}

As was said in the Introduction, analogously to the GO case, the term rGO covers a large class of polyderivatives of empty-core-based graphene molecules, with the only indication on the disposition of the chemical addends in the sheet circumference (ccf). A chemical composition of the relevant $\mathrm{rGO} c c f$ oxyhydride derivatives, as in the case of the GO ones, greatly varies depending on the rGO production protocols [6]. However, due to the fact that oxygens scatter neutrons inefficiently, the main contribution to the IINS of any rGO sample is provided with the vibrations that involve displacements of hydrogen atoms only. The hydrogens contribute into the rGO structure by the formation of $\mathrm{C}-\mathrm{H}$ bonds as well as of hydroxyls and carboxyls at the edge atoms. The presence of two latter addends depends on the rigidity of the performed reduction and can exist if only the reduction is not too hard [8]. In the case of hard reduction, the periphery of $\mathrm{rGO}$ molecules consists of $\mathrm{C}-\mathrm{H}$ and $\mathrm{C}=\mathrm{O}$ bonds only so that the IINS spectra of the samples are provided with hydrogens attached to the edge atoms. The reduction performed for the studied TErGO product is obviously hard, due to which, one can expect a pronounced similarity of the obtained $G(\omega)$ spectrum with those related to hard-reduced rGOs of different origin. 

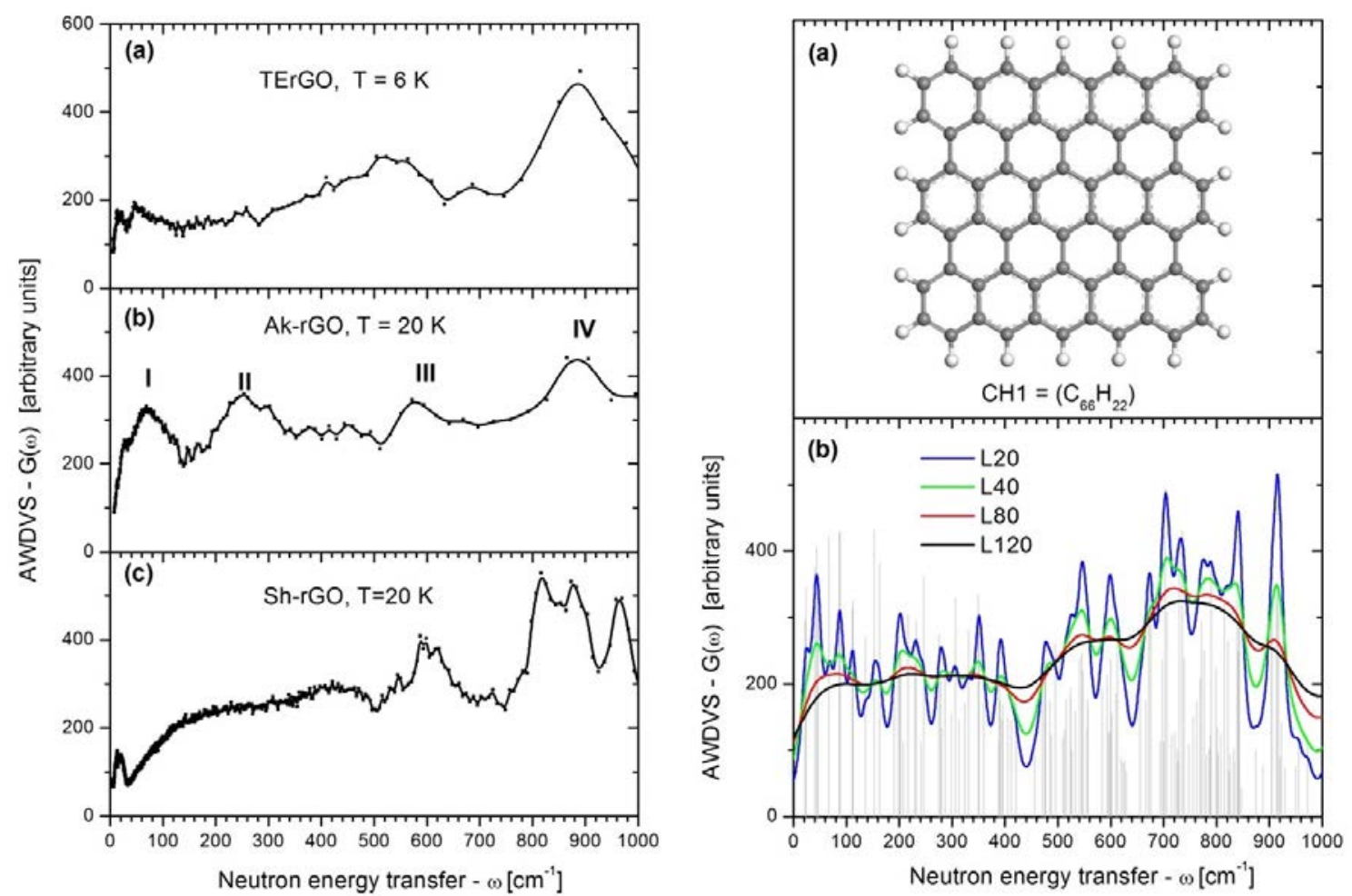

FIG. 8. AWDVS $G(\omega)$ spectra. Left: Experimental $G(\omega)$ spectra of TErGO at $6 \mathrm{~K} \mathrm{(a);} \mathrm{Ak-rGO} \mathrm{at} 20 \mathrm{~K}$ (b) [9], and sh-rGO at $20 \mathrm{~K}$ (c) [8]. Right. (a) Equilibrium structure and (b) computed $G(\omega)$ spectrum of $\mathrm{C}_{66} \mathrm{H}_{22}$ hydride. The 0-1 IINS vibrational mode intensities (gray bars) were convoluted by using Lorentzians of 20,40,80, and $120 \mathrm{~cm}^{-1}$ half width (blue, red, green, and black lines, respectively)

Two more such rGO samples have been studied by now: the first is synthetically produced Ak-rGO when using hydrogenolysis in supercritical isopropanol at the last stage on Scheme 1 [9]. The second sh-rGO presents shungite carbon of natural origin [8]. The obtained $G(\omega)$ spectra of the two samples are presented in the b and c left column panels of Fig. 8 . The spectrum of the TErGO studied in the current paper is presented in panel a. As seen in the figure, all the three spectra are evidently quite similar: all of them are spread over a large area from a few wavenumbers to more than thousand $\mathrm{cm}^{-1}$; there is a common feature of the spectra related to their most intensive part in the region of $800-1000 \mathrm{~cm}^{-1}$ (part IV); the spectra below $800 \mathrm{~cm}^{-1}$ look like a steady equal-intensity background, slightly structured: the amplitude of the structuring (parts I, II, and III) is comparable with the background intensity or less. The structuring is different for all the samples and presents that very mark that distinguish the latter. Therefore, contrary to the expectations of a close similarity, the spectra turned out rather different. This means that not only chemical composition of the $c c f$ area, but other factors also influence the IINS spectra image. Thus, features I and III in the spectrum of TErGO should be partially attributed to small amount of water in the sample (see Fig. 2b).

When examining the $G(\omega)$ spectrum of the Ak-GO [9], it was shown that the latter is fairly consistent with the calculated $G(\omega)$ spectrum of $c c f$ graphene hydrides. One such hydride, namely, $c c f(5,5)$ monohydride, is presented in the right a panel of Fig. 8 while its computed spectra is shown in panel b. As seen in the figure, the spectrum of vibrations, which involve displacements of hydrogen atoms, is very rich and spread over all the area 
where experimental spectra are observed. This very large spectrum width, which is a specific peculiarity of the spectrum, is provided by a peculiar dynamics of hydrogen atoms of the $c c f$ graphene hydrides due to participation of the atoms in all vibrational modes of the molecule. Part of these modes can be attributed to the vibrations related to framing $\mathrm{C}-\mathrm{H}$ bonds. Thus, the above mentioned feature IV centered at $900 \mathrm{~cm}^{-1}$ covers well characterized non-planar deformational vibrations of the bonds. This is the reason why it is observed in all the three experimental spectra. Vibrations below $800 \mathrm{~cm}^{-1}$ are mainly attributed to different modes with the dominant participation of carbon atoms. However, the eigenvectors of these vibrations involve hydrogen atoms, the displacement of which causes a peculiar 'riding effect' [19] due to which the vibrations of carbon atoms become vivid under neutron scattering. Conversely, the community of such carbon-dominated vibrations is evidently dependent on the size and shape of the rGO molecule, on the interaction between the rGO sheets and their number in the rGO stacks, on packing of the rGO stacks in massive samples, and so forth. Since the latter motifs are obviously different in the studied samples, the observed difference in the structured part of the $G(\omega)$ spectra below $800 \mathrm{~cm}^{-1}$ should be expected just revealing a structural polyvariance of rGO products thus exhibiting their topochemical nature.

\section{Concluding remarks}

The current paper brings to a close an extended neutron scattering study of a set of $\mathrm{GO}$ and rGO products of different origin. The first part concerned the $\mathrm{rGO}$ of natural origin presented by shungite carbon [8], the second was related to synthetic GO and rGO with the latter produced in the course of chemical treatment [9], and the current study was devoted to another pair of synthetic $\mathrm{GO} / \mathrm{rGO}$ products with the latter produced via thermal exfoliation of the parent GO. The study involved both the neutron diffraction (ND) and inelastic neutron scattering (INS). The obtained results gave convincing evidence for a significant polyvariance of both chemical composition and structural motifs that is the main peculiar characteristic of the products. Additionally, the results well demonstrated the ability of the neutron scattering to exhibit the polyvariance and provide means for its description at the microscopic level.

Briefly summarized, the obtained results for the studied GO and rGO may be presented as the following. For GOs, the neutron diffraction showed that the standardly pretreated fresh samples are characterized by an interlayer distance of $\sim 7 \AA$, which is characteristic for the product stored at ambient conditions under the lowest humidity. However, prolonged storage under more humid conditions causes an increase in the distance due to additional adsorption of water. INS from the samples is mainly provided by retained water and evidently shows the water interaction with the GO substrate resulting in the production of hydroxyls tightly bound with the carbon skeleton of the GO samples [9]. The water spectrum is noticeably different for the two samples indicating different arrangements of water molecules in the two cases. The latter is of the topochemical nature, once depending on both detail chemical composition of the GO surfaces as well as of size and shape of the GO sheets.

Neutron diffraction of the three rGO samples has exhibited a common feature related to the interlayer distance of $3.36-3.51 \AA$, thus indicating a flattening of the carbon skeletons of all the three samples. At the same time, the rGO layers form multi-fold stacks of different thicknesses: from 2 layers for Ak-rGO to $5-6$ layers of sh-rGO and $4-6$ layers in the case of the TErGO. The layer linear dimensions are different as well: from $\sim 1.5 \mathrm{~nm}$ for sh-rGO to a submicron size for both synthetic rGOs. Thus, the structural studies have revealed a significant polyvariance.

The INS studies have convincingly shown that all the samples are significantly hydrogenenriched and their IINS spectra are well reproduced by computational spectra related to graphene 
hydrides with empty basal plane and monoatomic-hydrogen framing of the edge atoms. The monoatomic-hydrogen motif is well revealed as a number of $\mathrm{C}-\mathrm{H}$ bonds with characteristic vibrational feature related to non-planar deformational vibrations in the region of $800-$ $1000 \mathrm{~cm}^{-1}$. The feature has been observed in the spectra of all the three samples. However, the energy region shown in the IINS spectra is much larger due to the very large number of vibrations related mainly to the carbon skeleton. The manifestation of these vibrations in the observed IINS spectra below $800 \mathrm{~cm}^{-1}$ is due to a specific 'riding effect' which is caused by the presence of hydrogen atoms with noticeable displacements in eigenvectors of the relevant vibrational modes. Obviously, this contribution should depend on the topology of the carbon skeletons due to which it is evidently different by shape and intensity in the observed IINS spectra.

Therefore, the performed study has convincingly shown that neutron scattering should be considered as a useful techniques for examining GO and rGO products. The technique has clearly distinguished GO and rGO products and well exhibited both common features and differences related to the members of both communities. If retained water in GOs and graphene-hydride nature of rGOs provide the commonality of dynamic properties within each of the community, the difference in the relevant sheet topology is responsible for a noticeable variability of the latter. The study has convincingly shown the topochemical nature of the variability, giving evidence for the topochemical nature of the discussed products.

\section{Acknowledgements}

This research was supported in part by PL-Grid Infrastructure (Grant ID: phd2014, phd2013). The calculations with use of DMOL3 were performed within Polish National Accelrys License (Mars Supercomputer, CYFRONET, AGH, Cracow). The financial support of Polish Government Plenipotentiary for JINR in Dubna, Russia (Grant No. 44/27-01-2015/7/1121/5) is also gratefully acknowledged.

\section{References}

[1] Novoselov K.S., Fal'ko V.I., Colombo L., et al. A roadmap for graphene. Nature, 2012, 490, P. $192-200$.

[2] Sheka E.F. The uniqueness of physical and chemical natures of graphene: their coherence and conflicts. Int. J. Quantum Chem., 2014, 114, P. 1079-1095.

[3] Ferari A.C., Bonaccorso F., Fal'ko V., et al. Science and technology roadmap for graphene, related twodimensional crystals, and hybrid systems. Nanoscale, 2015, 7, P. 4598-4810.

[4] Peplow M. The quest for supercarbon. Nature, 2013, 503, P. 327-329.

[5] Sheka E.F., Popova N.A. Molecular theory of graphene oxide. Phys. Chem. Chem. Phys., 2013, 15, P. 1330413322.

[6] Chua C.K., Pumera M. Chemical reduction of graphene oxide: a synthetic chemistry viewpoint. Chem. Soc. Rev., 2014, 43, P. 291-312.

[7] Zhao J., Liu L., Li F. Graphene oxide: Physics and applications. Springer, 2014, 154 p.

[8] Sheka E.F., Rozhkova N.N., Holderna-Natkaniec K., Natkaniec I. Neutron scattering study of reduced graphene oxide of natural origin. Nanosystems: Phys. Chem. Math., 2014, 5, P. 659-676.

[9] Natkaniec I., Sheka E.F., Druzbicki K., et al. Computationally supported neutron scattering study of parent and chemically reduced graphene oxide (Submitted).

[10] AkKoLab LLC, URL: www.akkolab.ru.

[11] Hummers W.S., Offeman R.E. Preparation of graphitic oxide. J. Am. Chem. Soc., 1958, 80, P. $1339-1339$.

[12] Krishnan D., Kim F., Luo J., et al. Energetic graphene oxide: Challenges and opportunities. Nano Today, 2012, 7, P. 137-152.

[13] Buchsteiner A., Lerf A., Pieper J. Water dynamics in graphite oxide investigated with neutron scattering. $J$. Phys. Chem. B, 2006, 110, P. 22328-22338.

[14] Natkaniec I., Chudoba D., Hetmańczyk L., et al. Parameters of the NERA spectrometer for cold and thermal moderators of the IBR-2 pulsed reactor. J. Phys.: Conf. Series, 2014, 554, P. 012002. 
[15] Opalev S.V., Belenkov E.A. Experimental research of graphite structure transformation at mechanical milling. Proceedings of Chelyabinsk Scientific Center, 3, P. 27-30 [in Russian].

[16] Sheka E., Popova N. Molecular theory of graphene oxide. Phys. Chem. Chem. Phys., 2013, 15, P. 1330413332.

[17] Kovalevski V.V., Buseck P.R., Cowley J.M. Comparison of carbon in shungite rocks to other natural carbons: An X-ray and TEM study. Carbon, 2001, 39, P. 243-56.

[18] Sofer Z., Šimek P., Jankovský O., et al. Neutron diffraction as a precise and reliable method for obtaining structural properties of bulk quantities of graphene. Nanoscale, 2014, 6, P. 13082-13089.

[19] Bokhenkov E.L., Natkaniec I., Sheka E.F. Determination of density of phonon states in a naphthalene crystal on basis of inelastic incoherent neutron scattering. Sov. Phys. JETP, 1976, 43, P. 536-545.

[20] Johnson M.R., Parlinski K., Natkaniec I., et al. Ab initio calculations and INS measurements of phonons and molecular vibrations in a model peptide compound - urea. Chem. Phys., 2003, 291, P. 53-60.

[21] Liao K.-H., Mittal A., Bose S., et al. Aqueous only route toward graphene from graphite oxide. ACS Nano, 2011, 5, P. 1253-1258.

[22] Dimiev A.M., Alemany L.B., Tour J.M. Graphene oxide. Origin of acidity, its instability in water, and a new dynamic structural model. ACS Nano, 2013, 7, P. 576-588.

[23] Sheka E., Popova N. Odd-electron molecular theory of graphene hydrogenation. J. Mol. Mod., 2012, 18, P. 3751-3768. 